Pacific Journal of Mathematics

THE RANGE OF A DERIVATION AND IDEALS 


\title{
THE RANGE OF A DERIVATION AND IDEALS
}

\author{
R. E. WEBER
}

When $A$ is in the Banach algebra $\mathscr{B}(\mathscr{C})$ of all bounded linear operators on a Hilbert space $\mathscr{H}$, the derivation generated by $A$ is the bounded operator $\Delta_{A}$ on $\mathscr{B}(\mathscr{C})$ defined by $\Delta_{A}(X)=A X-X A$. It is shown that the range of a derivation generated by a Hilbert-Schmidt or a diagonal operator contains no nonzero one-sided ideals of $\mathscr{B}(\mathscr{H})$. Also, for a two-sided ideal $\mathscr{J}$ of $\mathscr{B}(\mathscr{C})$, necessary and sufficient condition on an operator $A$ are given in order that the range of $\Delta_{A}$ equals the range of $\Delta_{A}$ restricted to $\mathscr{F}$.

1. In the following $\mathscr{H}$ will denote an infinite dimensional complex Hilbert space.

For a fixed $A \in \mathscr{B}(\mathscr{H})$, we will concern ourselves with the following problems:

(a) For what $B \in \mathscr{B}(\mathscr{H})$ is $B \mathscr{R}\left(\Delta_{A}\right) \subset \mathscr{R}\left(\Delta_{A}\right)$ or $\mathscr{R}\left(\Delta_{A}\right) B \subset$ $\mathscr{R}\left(\Delta_{A}\right)$.

(b) For what $B \in \mathscr{B}(\mathscr{H})$ is $B \mathscr{B}(\mathscr{H}) \subset \mathscr{R}\left(\Delta_{A}\right)$ or $\mathscr{B}(\mathscr{H}) B \subset$ $\mathscr{R}\left(\Delta_{A}\right)$.

(c) For what $B \in \mathscr{B}(\mathscr{H})$ is $\mathscr{R}\left(\Delta_{B}\right) \subset \mathscr{R}\left(\Delta_{A}\right)$.

It is easy to verify that for $A, X, Y \in \mathscr{B}(\mathscr{H})$.

and

(i ) $\Delta_{A}=\Delta_{A+\lambda}$ for all $\lambda \in \mathscr{C}$

(ii) $\Delta_{A}(X Y)=X \Delta_{A}(Y)+\Delta_{A}(X) Y$.

The identity (ii) yields some simple facts about the range of a derivation which show the interrelation of the above problems. (For a proof see [8].)

Lemma 1. Let $A, B \in \mathscr{B}(\mathscr{H})$ and let $A^{\prime}$ belong to the commutant $\{A\}^{\prime}$ of $A$. Then

(a) both $A^{\prime} \mathscr{R}\left(\Delta_{A}\right)$ and $\mathscr{R}\left(\Delta_{A}\right) A^{\prime}$ are contained in $\mathscr{R}\left(\Delta_{A}\right)$.

(b) if $\mathscr{R}\left(\Delta_{B}\right) \subset \mathscr{R}\left(\Delta_{A}\right)$, then both $\Delta_{A^{\prime}}(B) \mathscr{B}(\mathscr{H})$ and $\mathscr{B}(\mathscr{H}) \Delta_{A^{\prime}}(B)$ are contained in $\mathscr{R}\left(\Delta_{A}\right)$.

(c) $B \mathscr{R}\left(\Delta_{A}\right) \subset \mathscr{R}\left(\Delta_{A}\right)$ if and only if $\Delta_{A}(B) \mathscr{B}(\mathscr{H}) \subset \mathscr{R}\left(\Delta_{A}\right)$.

(d) $\mathscr{R}\left(\Delta_{A}\right) B \subset \mathscr{R}\left(\Delta_{A}\right)$ if and only if $\mathscr{B}(\mathscr{H}) \Delta_{A}(B) \subset \mathscr{R}\left(\Delta_{A}\right)$.

From (b) of Lemma 1 it follows that if $\mathscr{R}\left(\Delta_{A}\right)$ does not contain left- or right-ideals, then a necessary condition for $\mathscr{R}\left(\Delta_{B}\right) \subset \mathscr{R}\left(\Delta_{A}\right)$ is that $B \in\{A\}^{\prime \prime}$. In fact, more is true:

Lemma 2. Let $A \in \mathscr{B}(\mathscr{H})$. If $\mathscr{R}\left(\Delta_{A}\right)$ contains either no nonzero left-ideals or no nonzero right-ideals, then $\Delta_{B}(\mathscr{F}) \subset \mathscr{R}\left(\Delta_{A}\right)$ implies 
$B \in\{A\}^{\prime \prime} . \quad$ (F denotes the ideal of finite rank operators.)

Proof. Assume that $\mathscr{R}\left(\Delta_{A}\right)$ contains no nonzero left-ideals (the argument for the other assumption is similar). Let $P$ be a finite rank projection. If $A^{\prime} \in\{A\}^{\prime}$, then

$$
\Delta_{A^{\prime}}(B) P X=A^{\prime} \Delta_{B}(P X)-\Delta_{B}\left(A^{\prime} P X\right)
$$

is in $\mathscr{R}\left(\Delta_{A}\right)$ for all $X \in \mathscr{B}(\mathscr{H})$. Therefore, $\Delta_{A^{\prime}}(B) P \mathscr{B}(\mathscr{H}) \subset \mathscr{R}\left(\Delta_{A}\right)$ and hence $\Delta_{A^{\prime}}(B) P=0$. However, this is true for any such $P$ and hence $\Delta_{A^{\prime}}(B)=0$.

For the sake of completeness we include a somewhat simpler proof of a theorem of Stampfli [6]. In the proof, $\sigma_{l}(A)$ denotes the left essential spectrum of $A$ and is defined to be the set of those $\lambda$ for which the coset of the Calkin algebra $\mathscr{B}(\mathscr{C}) / \mathscr{K}$ (where $\mathscr{K}$ is the ideal of compact operators) containing $A-\lambda$ fails to have a left inverse. The right essential spectrum $\sigma_{r}(A)$ is defined in the obvious way.

THEOREM 1. Let $A \in \mathscr{B}(\mathscr{C})$. Then $\mathscr{R}\left(\Delta_{A}\right)$ contains no nonzero two-sided ideals of $\mathscr{B}(\mathscr{H})$.

Proof. Replace $A$ by $A-\lambda$ where $\lambda \in \sigma_{l}(A) \cap \sigma_{r}(A)$ if necessary in order to assume that there exist orthonormal sequences $\left\{f_{n}\right\}$ and $\left\{g_{n}\right\}$ such that $\sum\left\|A f_{n}\right\|^{1 / 2}<\infty$ and $\sum\left\|A^{*} g_{n}\right\|^{1 / 2}<\infty$. (See [6].) Then for all $X \in \mathscr{B}(\mathscr{C})$,

$$
\sum\left|\left((A X-X A) f_{n}, g_{n}\right)\right|^{1 / 2} \leqq \sum \| X||^{1 / 2}\left(\left\|A^{*} g_{n}\right\|^{1 / 2}+\left\|A f_{n}\right\|^{1 / 2}\right)<\infty .
$$

If $\mathscr{R}\left(\Delta_{A}\right)$ contains a two-sided ideal, then it contains all finite rank operators. In particular, if $f \otimes g$ denotes the rank one operator $f \otimes g(x)=(x, g) f$, then $(f \otimes f) X \in \mathscr{R}\left(\Delta_{A}\right)$ for all $f \in \mathscr{C}$ and $X \in \mathscr{B}(\mathscr{C})$. Hence

$$
\sum\left|\left((f \otimes f) X f_{n}, g_{n}\right)\right|^{1 / 2}<\infty .
$$

Since

$$
\begin{aligned}
\sum\left|\left((f \otimes f) X f_{n}, g_{n}\right)\right|^{1 / 2} & =\sum\left|\left(X f_{n},(f \otimes f) g_{n}\right)\right|^{1 / 2} \\
& =\sum\left|\left(X f_{n}, f\right)\left(\overline{g_{n}, f}\right)\right|^{1 / 2},
\end{aligned}
$$

then

$$
\sum\left|\left(X f_{n}, f\right)\left(\overline{g_{n}, f}\right)\right|^{1 / 2}<\infty
$$

for all $f \in \mathscr{H}$ and $X \in \mathscr{B}(\mathscr{H})$. However, if we choose $X$ such that $X f_{n}=g_{n}$ and $f$ such that $\left\{\left|\left(g_{n}, f\right)\right|\right\}$ is not summable, we have a contradiction. 
2. Let $\mathscr{S}$ denote the set of Hilbert-Schmidt operators on $\mathscr{H}$. Equipped with the trace inner product $(A, B)=\operatorname{tr}\left(A B^{*}\right), \mathscr{S}$ is a Hilbert space [5]. If $A \in \mathscr{B}(\mathscr{H})$, then the restriction of $\Delta_{A}$ to $\mathscr{S}$ is a bounded operator on $\mathscr{S}$ with adjoint $\left(\Delta_{A} \mid \mathscr{S}\right)^{*}=\Delta_{A^{*}} \mid \mathscr{S}$. Hence $\mathscr{S}=\mathscr{R}\left(\Delta_{A} \mid \mathscr{S}\right)^{=} \bigoplus\left(\left\{A^{*}\right\}^{\prime} \cap \mathscr{S}\right)$ where the double bar indicates closure with respect to the topology on $\mathscr{S}$.

\section{Theorem 2. Let $A \in \mathscr{S}$. Then $\mathscr{R}\left(\Delta_{A}\right)==\mathscr{R}\left(\Delta_{A} \mid \mathscr{S}\right)^{=}$.}

Proof. It follows from the above remarks that $\mathscr{R}\left(\Delta_{A}\right)^{\perp} \subset$ $\mathscr{R}\left(\Delta_{A} \mid \mathscr{S}\right)^{-}{ }^{*}=\left\{A^{*}\right\}^{\prime} \cap \mathscr{S}$. It remains to show the reverse inclusion. Let $T \in\left\{A^{*}\right\}^{\prime} \cap \mathscr{S}$. Then for $X \in \mathscr{B}(\mathscr{\mathscr { C }})$

$$
\begin{aligned}
\left(\Delta_{A}(X), T\right) & =\operatorname{tr}\left(T^{*} \Delta_{A}(X)\right)=\operatorname{tr}\left(T^{*} A X\right)-\operatorname{tr}\left(T^{*} X A\right) \\
& =\operatorname{tr}\left(A T^{*} X\right)-\operatorname{tr}\left(T^{*} X A\right)=\operatorname{tr}\left(T^{*} X A\right)-\operatorname{tr}\left(T^{*} X A\right)=0 .
\end{aligned}
$$

Therefore $T \in \mathscr{R}\left(\Delta_{A}\right)^{\perp}$.

Corollary. Let $A \in \mathscr{S}$. Then $\mathscr{R}\left(\Delta_{A}\right)=\oplus\left(\left\{A^{*}\right\}^{\prime} \cap \mathscr{S}\right)=\mathscr{S}$.

THEOREM 3. If $A \in \mathscr{S}$, then $\mathscr{R}\left(\Delta_{A}\right)$ does not contain any nonzero left- or right-ideals.

In the proof of Theorem 3 we will make use of the following result.

Lemma 3. Let $A \in \mathscr{S} . \quad$ If $(f \otimes f) \mathscr{B}(\mathscr{H}) \subset \mathscr{R}\left(\Delta_{A}\right)$, then $A f=0$.

Proof. Since $\mathscr{R}\left(\Delta_{A}\right) \perp\left\{A^{*}\right\}^{\prime} \cap \mathscr{S}$, then $0=\operatorname{tr}(A(f \otimes f) X)=$ $\operatorname{tr}\left(A f \otimes X^{*} f\right)=\left(A f, X^{*} f\right)$ for all $X \in \mathscr{B}(\mathscr{C})$. Hence $A f=0$.

Proof of Theorem 3. Suppose that $(f \otimes f) \mathscr{B}(\mathscr{H}) \subset \mathscr{R}\left(\Delta_{A}\right)$. Then $f \otimes f=\Delta_{A}(X)$ for some $X \in \mathscr{B}(\mathscr{H})$ and by Lemma $3, f=(f \otimes f) f=$ $A X f-X A f=A X f$. Since $(f \otimes f) \mathscr{B}(\mathscr{C})=\Delta_{A}(X) \mathscr{B}(\mathscr{C}) \subset \mathscr{R}\left(\Delta_{A}\right)$, then by Lemma 1, $X \mathscr{R}\left(\Delta_{A}\right) \subset \mathscr{R}\left(\Delta_{A}\right)$. Therefore, $((X f) \otimes(X f)) \mathscr{B}(\mathscr{H}) \subset$ $X(f \otimes f) \mathscr{B}(\mathscr{C}) \subset \mathscr{R}\left(\Delta_{A}\right)$ and by Lemma $3, X f \in \operatorname{ker}(A)$. Hence $f=A X f=0$. The remainder follows by taking adjoints.

Corollary 1. Let $A \in \mathscr{S}$ and $B \in \mathscr{B}(\mathscr{C})$. Then $B \mathscr{R}\left(\Delta_{A}\right) \subset$ $\mathscr{R}\left(\Delta_{A}\right)$ if and only if $B \in\{A\}^{\prime}$.

Proof. This follows from Lemma 1 and the theorem.

Corollary 2. Let $A \in \mathscr{S}$. If $\Delta_{B}(\mathscr{F}) \subset \mathscr{R}\left(\Delta_{A}\right)$ then $B \in\{A\}^{\prime \prime}$.

Proof. This follows from Lemma 2 and the theorem. 
3. We now turn our attention to diagonal operators. When expressing a diagonal operator as the sum $A=\sum \alpha_{n} P_{n}$, unless otherwise stated we shall assume that $P_{n}$ is the rank one projection onto the subspace spanned by $e_{n}$, where $\left\{e_{n}\right\}$ is an orthonormal basis. (However, we do not require that the $\alpha_{n}$ 's be distinct.) Each operator $X$ has a matrix $\left(x_{i j}\right)$ with respect to this fixed basis.

The principle result of this section is that the range of a derivation generated by a diagonal operator contains no nonzero left- or right-ideals. The theorem is slightly more general.

THEOREM 4. Let $A \in \mathscr{B}(\mathscr{C})$ have the property that there exist reducing subspaces $\mathscr{K}_{n}$ of $A$, each finite dimensional, such that $\mathscr{H}=\sum \oplus \mathscr{A}_{n}$. Then $\mathscr{R}\left(\Delta_{A}\right)$ contains no nonzero positive operators.

Proof. Let $P=\Delta_{A}(X)$ where $P$ is positive. If $P_{n}$ is the orthogonal projection onto $\mathscr{C}_{n}$, then $P_{n} P \mid \mathscr{M}_{n}=A_{n} X_{n}-X_{n} A_{n}$ where $A_{n}=$ $A \mid \mathscr{C}_{n}$ and $X_{n}$ is the compression of $X$ to $\mathscr{C}_{n}$. Since $\mathscr{C}_{n}$ is finite dimensional, then $\operatorname{tr}\left(P_{n} P \mid \mathscr{C}_{n}\right)=0$. Hence $P_{n} P \mid \mathscr{M}_{n}$ being a positive operator with zero trace, must be 0 . Therefore, $P_{n} P P_{n}=0$ (on $\mathscr{C}$ ). Hence $P^{1 / 2} P_{n}=0$ and $P^{1 / 2}=0$.

COROLlary 1. If $A$ satisfies the hypothesis of the theorem and if either $B \mathscr{R}\left(\Delta_{A}\right)$ or $\mathscr{R}\left(\Delta_{A}\right) B$ is contained in $\mathscr{R}\left(\Delta_{A}\right)$, then $B \in\{A\}^{\prime}$.

Corollary 2. If $A$ satisfies the hypothesis of the theorem and $\Delta_{B}(\mathscr{F}) \subset \mathscr{R}\left(\Delta_{A}\right)$, then $B \in\{A\}^{\prime \prime}$.

COROllary 3. Let $A$ be normal with finite spectrum. Then for $B \in \mathscr{B}(\mathscr{C}), \mathscr{R}\left(\Delta_{B}\right) \subset \mathscr{R}\left(\Delta_{A}\right)$ if and only if $B \in\{A\}^{\prime \prime}$.

Proof. If $B \in\{A\}^{\prime \prime}$ then $B$ is a polynomial of $A$ and hence $\mathscr{R}\left(\Delta_{B}\right) \subset$ $\mathscr{R}\left(\Delta_{A}\right)$. (See [1, p. 79].) The converse follows from Corollary 2.

Lemma 4. Let $A, B \in \mathscr{B}(\mathscr{H})$ where $A=\sum \alpha_{i} P_{i}$. Then $\mathscr{R}\left(\Delta_{B}\right) \subset$ $\mathscr{R}\left(\Delta_{A}\right)$ if and only if $B=\sum \beta_{i} P_{i}$ for some set of scalars $\beta_{0}, \beta_{1} \cdots$ and for every operator $X=\left(x_{i j}\right) \in \mathscr{B}(\mathscr{H})$ there exists an operator $Y=\left(y_{i j}\right) \in \mathscr{B}(\mathscr{C})$ such that $\left(\alpha_{i}-\alpha_{j}\right)=\left(\beta_{i}-\beta_{j}\right) x_{i j}$ for all $i, j$.

Proof. This follows from Corollary 2 and the fact that $\left[\Delta_{A}(X)\right]_{i j}=$ $\left(\alpha_{i}-\alpha_{j}\right) x_{i j}$ if $X=\left(x_{i j}\right)$.

Theorem 5. Let $A \in \mathscr{B}(\mathscr{H})$ be diagonal. If for $B \in \mathscr{B}(\mathscr{H})$, $\mathscr{R}\left(\Delta_{B}\right) \subset \mathscr{R}\left(\Delta_{A}\right)$, then $B=f(A)$ for some function $f$ which is Lipschitz on the spectrum of $A$. 
Proof. Let $A=\sum \alpha_{i} P_{i}$. If $\mathscr{R}\left(\Delta_{B}\right) \subset \mathscr{R}\left(\Delta_{A}\right)$, then by Corollary $2, B=\sum \beta_{i} P_{i}$ for some sequence of scalars $\left\{\beta_{i}\right\}$ and for any $X=$ $\left(x_{i j}\right) \in \mathscr{B}(\mathscr{C})$, there exists a $Y=\left(y_{i j}\right) \in \mathscr{B}(\mathscr{C})$ such that $y_{i j}=\left(\left(\beta_{i}-\right.\right.$ $\left.\left.\beta_{j}\right) /\left(\alpha_{i}-\alpha_{j}\right)\right) x_{i j}$ whenever $\alpha_{i} \neq \alpha_{j}$. It follows that $\left(\left(\beta_{i}-\beta_{j}\right) /\left(\alpha_{i}-\alpha_{j}\right)\right)$ is bounded by some positive number $M$. Define $f$ such that $f\left(\alpha_{i}\right)=\beta_{i}$. Then $f$ is a Lipschitz function defined on a dense subset of $\sigma(A)$ onto a dense subset of $\sigma(B)$. Therefore, we can extend $f$ to be Lipschitz on $\sigma(A)$ onto $\sigma(B)$.

It was shown in [7] that if $B$ is an analytic function of $A$, then $\mathscr{R}\left(\Delta_{B}\right) \subset \mathscr{R}\left(\Delta_{A}\right)$. To have range inclusion it is neither necessary that $B$ be an analytic function of $A$ nor sufficient that $B$ be a continuous function of $A$ as seen in the next two examples.

Example 1. Let $A=\sum \alpha_{n} P_{n}$ where $\operatorname{dim} P_{n}=1, \alpha_{0}=0$, and

$$
\alpha_{n}=\left\{\begin{array}{llll}
i / n & \text { for } & n & \text { even } \\
1 / n & \text { for } & n & \text { odd }
\end{array}\right.
$$

Let $B=\sum \beta_{n} P_{n}$ where $\beta_{0}=0$ and $\beta_{n}=-i / n^{2}$ for $n \geqq 1$. A direct computation shows that if $n<m$, then $\left|\left(\beta_{n}-\beta_{m}\right) /\left(\alpha_{n}-\alpha_{m}\right)\right| \leqq 2 / n$. Now, for any $X=\left(x_{i j}\right) \in \mathscr{B}(\mathscr{\mathscr { C }})$, consider the matrix $Y=\left(y_{i j}\right)$ where $y_{i j}=\left(\left(\beta_{i}-\beta_{j}\right) /\left(\alpha_{i}-\alpha_{j}\right)\right) x_{i j}$ whenever $\alpha_{i} \neq \alpha_{j}$ and zero otherwise. Then

$$
\sum_{i, j}\left|y_{i j}\right|^{2}=\sum_{n=0}^{\infty} \sum_{j=n}^{\infty}\left|y_{n j}\right|^{2}+\sum_{m=0}^{\infty} \sum_{i=m}^{\infty}\left|y_{i m}\right|^{2}
$$

For $m>0$,

$$
\sum_{i=m}^{\infty}\left|y_{i m}\right|^{2} \leqq 4 / m^{2} \sum_{i=m}^{\infty}\left|x_{i m}\right|^{2} \leqq 4 / m^{2}\|X\|^{2}
$$

and for $n>0$,

$$
\sum_{j=n}^{\infty}\left|y_{n j}\right|^{2} \leqq 4 / n^{2}\|X\|^{2}
$$

Hence

$$
\sum_{i, j}\left|y_{i j}\right|^{2} \leqq\|X\|^{2}+\sum_{m=1}^{\infty} 4 / n^{2}\|X\|^{2}+\|X\|^{2}+\sum_{m=1}^{\infty} 4 / m^{2}\|X\|^{2} .
$$

Therefore, $Y \in \mathscr{B}(\mathscr{H})$ and by Lemma 4, $\mathscr{R}\left(\Delta_{B}\right) \subset \mathscr{R}\left(\Delta_{A}\right)$. Now, assume $f$ is an analytic function on $\sigma(A)$ such that for even $n, f(i / n)=$ $-i / n^{2}$. Then $f(z)=z^{2} i$. Hence for odd $n, f(1 / n)=i / n^{2} \neq-i / n^{2}$ and $B \neq f(A)$.

ExAMPle 2. Let $A=\sum \alpha_{n} P_{n}$ where $P_{n}$ is rank one for all $n$, $\alpha_{0}=0$, and $\alpha_{n}=1 / n^{2}$ for $n>0$ and let $B=\sum \beta_{n} P_{n}$ where $\beta_{0}=0$ 
and $\beta_{n}=1 / n$ for $n>0$. Then $B$ is a continuous function of $A$, in fact $B=f(A)$ where $f(z)=z^{1 / 2}$. Let $X=\left(x_{i j}\right) \in \mathscr{B}(\mathscr{C})$ where

$$
x_{n j}=\left\{\begin{array}{l}
1 / n \text { for } n>0 \text { and } j=0 \\
0 \text { otherwise }
\end{array}\right.
$$

If $\Delta_{B}(X)=\Delta_{A}(Y)$ where $Y=\left(y_{i j}\right)$, then

$$
y_{n 0}=x_{n 0}\left(\beta_{n}-\beta_{0}\right) /\left(\alpha_{n}-\alpha_{0}\right)=(1 / n)(1 / n) /\left(1 / n^{2}\right)=1
$$

for all $n$. Hence $Y \notin \mathscr{B}(\mathscr{H})$ and $\mathscr{R}\left(\Delta_{B}\right) \not \subset \mathscr{R}\left(\Delta_{A}\right)$.

Other derivations whose ranges do not contain any nonzero onesided ideals are those generated by unitary and self-adjoint operators. (See [9].)

It was shown in [7] that the range of a derivation generated by a nonunitary isometry does contain nonzero left-ideals. Other operators which possess this property are some of the weighted shifts.

4. Another question concerning the range of a derivation and, in this case, a two-sided ideal $\mathscr{F}$ of $\mathscr{B}(\mathscr{H})$ is whether $\mathscr{R}\left(\Delta_{A}\right)=\Delta_{A}(\mathscr{I})$.

THeOREM 6. Let $A \in \mathscr{B}(\mathscr{C})$ and let $\mathscr{J}$ be a proper two-sided ideal of $\mathscr{B}(\mathscr{H})$. Consider the following conditions:

(a) $\{A\}^{\prime}+\mathscr{J}=\mathscr{B}(\mathscr{H})$.

(b) $\mathscr{R}\left(\Delta_{A}\right)=\Delta_{A}(\mathscr{F})$.

(c) $\mathscr{R}\left(\Delta_{A}\right) \subset \mathscr{J}$.

(d) $A=T-\lambda$ for some $T \in \mathscr{F}$ and $\lambda \in \mathscr{C}$.

(a) is equivalent to (b), (c) is equivalent to (d), and (b) implies (c).

Proof. That (a) is equivalent to (b) is a consequence of the fact that $X=T+A^{\prime}$ for some $T \in \mathscr{J}$ and $A^{\prime} \in\{A\}^{\prime}$ if and only if $\Delta_{A}(X) \in$ $\Delta_{A}(\mathscr{J})$. That (c) is equivalent to (d) is a consequence of a theorem of Calkin [2] where he shows that the center of $\mathscr{B}(\mathscr{C}) / \mathscr{F}$ consists of scalars. It is immediate that (b) implies (c).

REMARK. An example to show that (c) does not imply (b) for the case when $\mathscr{F}$ is the ideal of compact operators can be obtained by letting $A$ be the adjoint of the weighted shift with weights $\{2,1$, $1 / 2,1 / 3, \cdots\}$ and showing that each element of $\{A\}^{\prime}$ is the translate of a Hilbert-Schmidt operator. (See [8].)

If we require only that the closures be equal, we have the following;

THEOREM 7. Let $A \in \mathscr{B}(\mathscr{H})$ be compact and let $\mathscr{F}$ be the ideal of finite rank operators. Then $\mathscr{R}\left(\Delta_{A}\right)^{-}=\Delta_{A}(\mathscr{F})^{-}$. 
Proof. Let $f \in \mathscr{B}(\mathscr{P})^{*}$. Then $f=f_{0}+f_{T}$ for some trace-class operator $T$ where $f_{T}(X)=\operatorname{tr}(X T)$ and where $f_{0}$ annihilates the compact operators. (See Dixmier [3].) If $f$ annihilates $\Delta_{A}(\mathscr{F})$ then $f_{T}\left(\Delta_{A}(F)\right)=f\left(\Delta_{A}(F)\right)=0$ for all $F \in \mathscr{F}$. However,

$$
\begin{aligned}
f_{T}\left(\Delta_{A}(F)\right) & =\operatorname{tr}((A F-F A) T)=\operatorname{tr}(A F T-F A T) \\
& =\operatorname{tr}(F T A-F A T)=\operatorname{tr}\left(F \Delta_{A}(-T)\right)
\end{aligned}
$$

for all $F \in \mathscr{F}$. Since $\mathscr{F}$ is dense in the trace-class operators, then $\Delta_{A}(-T)=0$ and $T \in\{A\}^{\prime}$. Hence $f_{T}$ annihilates the range of $\Delta_{A}$ and since $A$ is compact, $f\left(\Delta_{A}(X)\right)=f_{T}\left(\Delta_{A}(X)\right)=0$ for all $X \in \mathscr{B}(\mathscr{C})$.

If $A$ is normal then Theorem 6 can be improved;

THEOREM 8. Let $A \in \mathscr{B}(\mathscr{C})$ be normal and let $\mathscr{J}$ be a proper two-sided ideal of $\mathscr{B}(\mathscr{L})$. The following are equivalent:

(a) $\{A\}^{\prime}+\mathscr{J}=\mathscr{B}(\mathscr{C})$.

(b) $\mathscr{R}\left(\Delta_{A}\right)=\Delta_{A}(\mathscr{J})$.

(c) $\mathscr{R}\left(\Delta_{A}\right) \subset \mathscr{J}$ and $\sigma(A)$ is finite.

(d) $A=T-\lambda$ for some $T \in \mathscr{F}$, some $\lambda \in \mathscr{C}$ and $\sigma(A)$ is finite.

Proof. That (a) is equivalent to (b) and (c) is equivalent to (d) follows from Theorem 6 . If $A$ is normal with finite spectrum, then by a theorem of Anderson [1, p. 96] $\mathscr{R}\left(\Delta_{A}\right)+\{A\}^{\prime}=\mathscr{B}(\mathscr{H})$. Hence, if $A=T-\lambda$ for some $T \in \mathscr{J}$ and $\lambda \in \mathscr{C}$ then $\mathscr{R}\left(\Delta_{A}\right) \subset \mathscr{F}$ and (d) implies (a). To show that (a) implies (d), assume that $\sigma(A)$ is infinite and that $\{A\}^{\prime}+\mathscr{J}=\mathscr{B}(\mathscr{\mathscr { C }})$. Then by Theorem $6, A-\lambda \in \mathscr{J}$ for some $\lambda \in \mathscr{C}$. Since $\mathscr{F}$ is contained in the ideal of compact operators, we can assume that $A$ is compact. Let $A=A_{1} \oplus A_{2}$ on $\mathscr{M} \oplus \mathscr{H}^{\perp}$ where $A_{1}$ is an infinite dimensional diagonal operator with distinct eigenvalues and let $P$ be the orthogonal projection onto $\mathscr{A}$. Hence, if $X \in\{A\}^{\prime}$, then $P X P$ is diagonal. However, if we let $U$ be the unilateral shift on $\mathscr{C}$, then $\{A\}^{\prime}+\mathscr{F}=\mathscr{B}(\mathscr{H})$ implies that $U=$ $D+K$ for some diagonal operator $D$ and some compact operator $K$. This is clearly a contradiction (let $\left\{e_{n}\right\}$ be an orthonormal basis for $\mathscr{C l}$ by which $U$ is the shift, then $\left((D-U) e_{n}, e_{n+1}\right)=1$ for all $\left.n\right)$.

\section{REFERENCES}

1. J. H. Anderson, Derivations, Commutators, and The Essential Numerical Range, Thesis, Indiana University, 1971.

2. J. W. Calkin, Two-sided ideals and congruences in the ring of bounded operators in Hilbert space, Ann. of Math., 42 (1941), 839-872.

3. J. Dixmier, Les fonctionnelles linéaires sur l'ensemble des opératures bornés d'un espace de Hilbert, Ann. of Math., 51 (1950), 387-408.

4. R. G. Douglas, On majorization, factorization, and range inclusion of operators in Hilbert space, Proc. Amer. Math. Soc., 17 (1966), 413-416. 
5. R. Schatten, Norm Ideals of Completely Continuous Operators, 2nd printing, Ergebnisse der Mathematik und ihrer Grenzgebiete Band 27, Springer-Verlag, Berlin, 1970.

6. J. G. Stampfli, On the range of a derivation, Proc. Amer. Math. Soc., 40 (1973), 492-496.

7. R. E. Weber, Analytic functions, ideals, and derivation ranges, to appear.

8. — Derivation Ranges, Thesis, Indiana University, 1972.

9. J. P. Williams, On the range of a derivation $I I$, to appear.

Received November 28, 1972 and in revised form October 10, 1973. This paper contains part of a doctoral dissertation written under the direction of Professor J. P. Williams at Indiana University.

INDIANA UNIVERSITY SOUTHEAST 


\section{PACIFIC JOURNAL OF MATHEMATICS}

\section{EDITORS}

RICHARD ARENS (Managing Editor)

University of California

Los Angeles, California 90024

\section{R. A. Beaumont \\ University of Washington \\ Seattle, Washington 98105}

\section{J. DugundjI*}

Department of Mathematics University of Southern California Los Angeles, California 90007

D. Gilbarg and J. Milgram

Stanford University

Stanford, California 94305

\section{ASSOCIATE EDITORS}
E. F. BeCKenBaCH
B. H. NeumanN
F. WOLF
K. YoSHIDA

\section{SUPPORTING INSTITUTIONS}

\author{
UNIVERSITY OF BRITISH COLUMBIA \\ CALIFORNIA INSTITUTE OF TECHNOLOGY \\ UNIVERSITY OF CALIFORNIA \\ MONTANA STATE UNIVERSITY \\ UNIVERSITY OF NEVADA \\ NEW MEXICO STATE UNIVERSITY \\ OREGON STATE UNIVERSITY \\ UNIVERSITY OF OREGON \\ OSAKA UNIVERSITY
}

\author{
UNIVERSITY OF SOUTHERN CALIFORNIA \\ STANFORD UNIVERSITY \\ UNIVERSITY OF TOKYO \\ UNIVERSITY OF UTAH \\ WASHINGTON STATE UNIVERSITY \\ UNIVERSITY OF WASHINGTON

$* * *$
$*$
AMERICAN MATHEMATICAL SOCIETY
NAVAL WEAPONS CENTER

The Supporting Institutions listed above contribute to the cost of publication of this Journal, but they are not owners or publishers and have no responsibility for its content or policies.

Mathematical papers intended for publication in the Pacific Journal of Mathematics should be in typed form or offset-reproduced, (not dittoed), double spaced with large margins. Underline Greek letters in red, German in green, and script in blue. The first paragraph or two must be capable of being used separately as a synopsis of the entire paper. Items of the bibliography should not be cited there unless absolutely necessary, in which case they must be identified by author and Journal, rather than by item number. Manuscripts, in duplicate if possible, may be sent to any one of the four editors. Please classify according to the scheme of Math. Rev. Index to Vol. 39. All other communications to the editors should be addressed to the managing editor, or Elaine Barth, University of California, Los Angeles, California, 90024.

100 reprints are provided free for each article, only if page charges have been substantially paid. Additional copies may be obtained at cost in multiples of 50 .

The Pacific Journal of Mathematics is issued monthly as of January 1966. Regular subscription rate: $\$ 60.00$ a year (6 Vols., 12 issues). Special rate: $\$ 30.00$ a year to individual members of supporting institutions.

Subscriptions, orders for back numbers, and changes of address should be sent to Pacific Journal of Mathematics, 103 Highland Boulevard, Berkeley, California, 94708.

PUBLISHED BY PACIFIC JOURNAL OF MATHEMATICS, A NON-PROFIT CORPORATION

Printed at Kokusai Bunken Insatsusha (International Academic Printing Co., Ltd.), 270, 3-chome Totsuka-cho, Shinjuku-ku, Tokyo 160, Japan

* C. R. DePrima California Institute of Technology, Pasadena, CA 91109, will replace J. Dugundji until August 1974. 


\section{Pacific Journal of Mathematics}

Vol. 50, No. $2 \quad$ October, 1974

Mustafa Agah Akcoglu, John Philip Huneke and Hermann Rost, A counter example to the Blum Hanson theorem in general spaces .............

Huzihiro Araki, Some properties of modular conjugation operator of von

Neumann algebras and a non-commutative Radon-Nikodym theorem

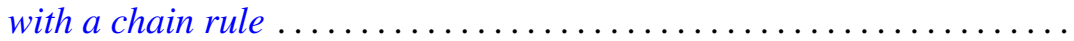

E. F. Beckenbach, Fook H. Eng and Richard Edward Tafel, Global properties of rational and logarithmico-rational minimal surfaces .....

David W. Boyd, A new class of infinite sphere packings ............. 383

K. G. Choo, Whitehead Groups of twisted free associative algebras ........

Charles Kam-Tai Chui and Milton N. Parnes, Limit sets of power series

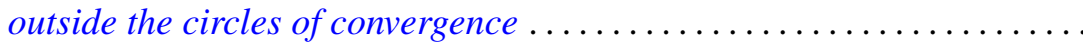

Allan Clark and John Harwood Ewing, The realization of polynomial

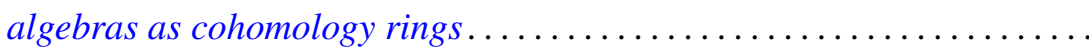

Dennis Garbanati, Classes of circulants over the p-adic and rational

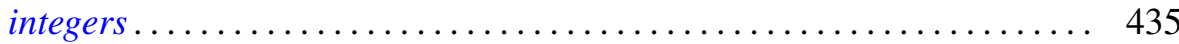

Arjun K. Gupta, On a "square" functional equation ................... 449

David James Hallenbeck and Thomas Harold MacGregor, Subordination and extreme-point theory ............................. 455

Douglas Harris, The local compactness of $v X \ldots \ldots . . . . . . . . . . . .4469$

William Emery Haver, Monotone mappings of a two-disk onto itself which fix the disk's boundary can be canonically approximated by homeomorphisms .................................. 477

Norman Peter Herzberg, On a problem of Hurwitz .................. 485

Chin-Shui Hsu, A class of Abelian groups closed under direct limits and subgroups formation ............................... 495

Bjarni Jónsson and Thomas Paul Whaley, Congruence relations and multiplicity types of algebras.....................

Lowell Duane Loveland, Vertically countable spheres and their wild sets.

Nimrod Megiddo, Kernels of compound games with simple components ....

Russell L. Merris, An identity for matrix functions ........ . .

E. O. Milton, Fourier transforms of odd and even tempered distributions ...

Dix Hayes Pettey, One-one-mappings onto locally connected generalized continua

Mark Bernard Ramras, Orders with finite global dimension

Doron Ravdin, Various types of local homogeneity. .

George Michael Reed, On metrizability of complete Moore spaces ...

Charles Small, Normal bases for quadratic extensions ..

Philip C. Tonne, Polynomials and Hausdorff matrices.... . . 